\title{
Perceptions and Behavioral Adoptions of the Preventive Strategies from Person-to-Person Transmission of COVID-19 among the Public in China: An Online Cross-Sectional Survey
}

\section{Xian Zhu}

Chongqing Medical University https://orcid.org/0000-0003-0254-5349

\section{Yong Gan}

Huazhong University of Science and Technology Tongji Medical College

Bo Wu

Wanzhou District Center for Disease Control and Prevention

\section{Qinyong Gou}

Nan'an Women and Children Health Hospital

\section{Daikun Zheng}

Chongqing Three Gorges Medical College

Chenglu Liu

Chongqing Medical University

Chang Xu

Chongqing Medical University

\section{Zhou Tao}

Chongqing Medical University

Hong Xu ( $\nabla$ xuhong@cqmu.edu.cn )

Chongqing Medical University

Qian Long

Duke Kunshan University

\section{Research Article}

Keywords: COVID-19, individual belief, behavioral adoption, health belief model

Posted Date: August 10th, 2020

DOl: https://doi.org/10.21203/rs.3.rs-54686/v1

License: (c) (1) This work is licensed under a Creative Commons Attribution 4.0 International License. 
Page 2/14 


\section{Abstract}

Background: The strategies adopted to prevent spreading of COVID-19 are quarantine, social distancing and isolation of infected cases. This study investigates perceptions and behavioral adoptions of COVID19 prevention strategies among the Chinese public and identified factors predicting individual health behavior.

Methods: We conducted a cross-sectional online survey between 22 February and $5 \mathrm{March}, 2020$. We approached to urban residents aged over 18 years through snowball sampling method using the Chinese social media. The Health Belief Model was adopted to guide the analysis. Bivariate and multivariate logistic regressions were used to examine impacts of modifying factors (including demographic and socio-economic characteristics) and individual beliefs on individual health behavior.

Results: of 5675 valid questionnaires, $95.8 \%$ of the respondents well understood the preventive measures from COVID-19 transmission, while $79.9 \%$ of the respondents adopted the behavior advised. $45.7 \%$ of the respondents perceived severity of the disease, $75.6 \%$ of the respondents perceived benefits of social constraints measures and $62.7 \%$ reported anxiety during the epidemic. After adjusting for modifying factors and individual beliefs, those who were female, had better income and good knowledge on preventive measures, perceived benefits on social constraint measures and did not feel anxiety were more likely to adopt behaviors advised.

Conclusions: The Chinese public highly accepted and adopted behaviors advised to slow down the COVID-19 epidemic. People with low income or feeling anxiety were less likely to adopt the behavior advised. The policy support should target on the social vulnerable groups. The psychological support should be disseminated through different means, and the consultation should be provided to those who are in need.

\section{Background}

Coronavirus disease 2019 (COVID-19) is a new respiratory infectious disease and has spreading globally. Up to Jun 2020, 215 countries have reported COVID-19 cases, more than 10 million confirmed cases and 500,000 deaths in the world. ${ }^{[1]}$ Currently, the most common transmission routes include droplet and aerosol transmission through the respiratory tract, close contact transmission, fecal-oral transmission. ${ }^{[2-}$ 3]

China is the first country witnessed COVID-19 outbreak and facing challenges of grappling with the scope and nature of both physical and mental health impacts. China had implemented stringent measures including quarantine, keep social distancing and isolation of infected cases to slow down COVID-19 epidemic. ${ }^{[4-5]}$ All provinces of Chinese mainland launched the highest level of emergency public health response by the end of January 2020. Self-protection measures, such as face masks, hands wash, indoor ventilation, respiratory hygiene habits, and social distancing were advocated and disseminated through 
the official public media, social media and community-based approaches ${ }^{[6-7]}$ How individuals respond to advice on how best to prevent transmission is critical, as currently there are lack of effective antiviral drugs and vaccine. ${ }^{[8-10]}$

The previous studies on the public responses to emerging infectious diseases reported poor adherence to the recommendations, although knowledge of the disease prevention among public showed relatively high. ${ }^{[1-12]}$ In Korea, around half of the study participants adhere to suggestions to preventing Middle East Respiratory Syndrome (MERS). ${ }^{[12]}$ In the Netherlands, the online survey with the general population found that $95 \%$ of the respondents had good knowledge of the Influenza A (H1N1), but only $36 \%$ of the respondents had taken preventive measures recommended. ${ }^{[11]}$ In this study, the older age, high perceived severity, high anxiety and high perceived efficacy of measures were positively associated with the behavior adoption. ${ }^{[11]}$

The Health Belief Model (HBM) has been widely used in health behavior research to guide interventions for health behavior change. ${ }^{[13-16]}$ The HBM has three main components: modifying factors, individual factors and action. ${ }^{[17]}$ Modifying factors include demographic and socio-economic characteristics and knowledge on disease. Individual factors refer to perceived severity, susceptibility, benefits, barriers and self-efficacy. Modifying factors impact on individual beliefs, and the combination of beliefs further affects individual action. According to the conceptual framework of the HBM, this study investigated perceptions and behavioral adoptions of the preventive strategies from person-to-person transmission of COVID-19 among the public in China and identified factors predicting individual health behavior.

\section{Methods}

\section{Methods and data collection}

The target population of this study was Chinese urban residents aged over 18 years who were not diagnosed as COVID-19. We conducted the online survey between 22 February and 5 March, 2020. We approached to the target population using snow-ball sampling method through the Chinese social media, Wechat and Weibo. The information was disseminated through two networks: 1) social media of medical students and faculty in Tongji Medical College in Wuhan and Chongqing Medical University in Chongqing; 2) social media of health professional at Wanzhou District Centre for Disease Control and Prevention (CDC) in Chongqing.

The research team developed the questionnaire based on the literature review and collected public opinions. The questionnaire was finalized after pilots. The questionnaire included several sections and had questions on personal and household demographic and socio-economic characteristics, individual knowledge on COVID-19 prevention, individual beliefs and personal response to advised strategies. The link of online survey was shared through social media. Each IP address is allowed to can only fill out the questionnaire once. The research team received a total of 5819 responses. Given the quality of the response, we excluded the questionnaires filled within 2 minutes. We also checked completeness of each 
questionnaire. After data cleaning, total 5675 (97.5\%) questionnaires were valid that were from 31 provinces (Fig. 1) of Chinese mainland and 240 cities and included in the analysis.

\section{Data analysis}

We developed the analysis framework, and studied the relationship between modifying factors and individual beliefs, and how they further predicted individual behaviors (Fig. 2).

Modifying factors included participant's sex, age, educational attainment (junior and senior high school and below, college, university and above), occupation (health professional, government or public institution staff, factory worker, students (both medical students and non-medical students) and others (including self-employed persons, retired person and unemployed)), annual household income (lower, lower middle, upper middle and upper), having family member who was health professional (yes, no), living area at risk level of COVID-19 prevalence (high, middle, low) and knowledge on preventive measures. Annual household income category was generated by the inter-quartile range, lower income group having less than $\$ 5143$ annually, lower middle group at the range of $\$ 5143 \sim \$ 8570$ annual income, upper middle group at the range of $\$ 8571 \sim \$ 17141$ annual income and was defined as upper middle group having more than $\$ 17,141$ annually. We defined the risk level of living areas according to the number of COVID-19 cases diagnosed. Hubei Province was the center of COVID-19 outbreak and referred to high-risk epidemic area; outside Hubei province, the area with the diagnosed cases more than 100 referred to middle-risk epidemic area, and the area with less than 100 cases referred to low-risk epidemic area. We asked five questions on face mask, hand wash, indoor ventilation, respiratory hygiene habits and social distancing. If participants answered all five questions correctly, they were grouped into "well known" preventive measures.

We measured three domains of individual beliefs: perceived severity, perceived benefits and perceived anxiety. We asked a question "Do you feel severity of the disease when you hear the following event: 1) the first death caused by COVID-19 announced by the Wuhan Health Committee; 2) "human-to-human" transmission confirmed and announced." If participants answered "yes" either of them, we grouped them into "perceived severity". In addition, we asked a question "what measures you think would be beneficial in your living area?" There were three alternatives: "a) mandatory wearing face mask when going out; $b$ ) suspending operation of public transportation; c) community lock-down". If participants ticked all three, we grouped them into "perceived benefits". We also asked "do you feel anxiety during the epidemic of COVID-19?" If participants answered "yes", we grouped them into "perceived anxiety".

We examined participant's adoptions of advised behaviors. We asked five questions: after the COVID-19 outbreak, 1) do you increase the frequency and length of hand wash? 2) do you keep indoor ventilation for more than 2 hours per day? 3) do you cover your mouth and nose with a tissue when coughing/ sneezing, then throw away the tissue and wash your hands? 4) do you keep away from people when you went out or never went out? 5) do you wear face mask when you go out? Every question could be answered "yes or no". If participants answered "yes" for all five questions, we grouped them into "full adoption" category. Otherwise, they were grouped into "partial adoption". 
Descriptive analysis was used to present modifying factors, individual beliefs and adoptions of advised preventive measures. We examined the relationship between modifying factors and individual beliefs using bivariate logistic regression analysis. Multivariate logistic regression analyses were used to examine association between modifying factors and individual beliefs and behavior adoptions adjusting for all modifying factors and three domains of individual beliefs, respectively. We used SPSS21.0 statistical software for statistical analysis. $P<0.05$ was considered statistically significant.

\section{Results}

\section{Modifying factors: demographic and socio-economic characteristics and knowledge on preventive measures}

Of 5675 participants, most were female accounting for $65.3 \%$. Mean age of the participants was 36 years old ranging from 18 years old to 85 years old. Around half of the respondents received university and above education. In addition, $14.1 \%$ of the respondents were health professionals and $36.0 \%$ of the respondents reported having health professional in the family. The few $(4.1 \%)$ respondents lived in the high-risk epidemic area and $38.8 \%$ of the respondents lived in the middle-risk epidemic area. A vast majority of the respondents (95.8\%) well understood COVID-19 relevant protection measures (Table 1).

\section{Individual beliefs and behavioral adoptions of advised measures}

Around half of the respondents (45.7\%) reported perceived severity of COVID-19 epidemic. Most of them (75.6\%) thought suspending operation of public transportation, mandatory wearing face mask when going out and community lock-down would have benefits to slow down epidemic of COVID-19. More than half $(62.7 \%)$ of the respondents reported anxiety during the epidemic of COVID-19. A vast majority of the respondents $(79.9 \%)$ adopted the all five basic protection measures advised. Of which, the acceptance of wearing face mask when going out was the highest (98.5\%), followed by increase of the frequency and length of hand wash (97.6\%), having good respiratory hygiene habits (93.6\%) and keeping indoor ventilation for more than 2 hours per day (93.3\%). Comparing to these measures, the proportion of keeping social distance was relatively low that was $87.1 \%$ (Table 2).

\section{Influencing factors of individual beliefs in the Chinese public}

We studied the association between individual beliefs and modifying factors using bivariate logistic regression analysis. Compared to male, the female respondents were more likely to perceive severity of the disease, perceive benefits of social restriction measures and feel anxiety during the epidemic of COVID-19. Young and middle age respondents did not feel much severity of the disease than the respondents aged over 50 years, but they were more likely to perceive benefits of the social restriction measures and feel anxiety than the older respondents. We found similar perceptions among the 
respondents with high education attainment and upper level annual household income. The respondents who were health professional or family member of health professional and those living in middle and high risk areas of COVID-19 prevalence were more likely to perceive benefits of social restriction measures and also feel anxiety during the period. Those who well understood all five basic protection measures advised did feel severity of the disease and benefits of social restriction measures, but the perceived anxiety was not significant (Table 3 ).

\section{Influencing factors of behavioral adoptions in the Chinese public}

According to the bivariate analysis, the respondents who were female, aged at 30-49 years, had college education, had better income and lived in areas at the middle level risk of the epidemic were more likely to take all five protection measures advised. Those who were health professional or family member of health professional had better behavior adoption than others. Moreover, those who had good knowledge on the protection measures were more likely to accept and adopt to the advices. In terms of individual belief, those who perceived severity and benefits were more likely to take measures advised (Table 4).

After adjusting for modifying factors, female and those who had better income were more likely to follow the measures advised. Compared to health professionals, factory workers and students were less likely to adopt to the measures advised. Knowledge on the protection measures were strongly associated with behavior adoption. After adjusting for individual beliefs, perceived severity and benefits were significantly associated with behavior adoption. After adjusting for modifying factors and individual beliefs at the same time, we found very similar results, but the association between perceived severity and behavior adoption was not statistically significant. Those who did not feel anxiety were positively associated with the behavior adoption (Table 4).

\section{Discussion}

This study found a vast majority of the public knew relevant preventive measures during the period of COVID-19 outbreak in China and most of them adopt preventive behavior according to the advices. Those who were female, young and middle age, had high education attainment and better annual household income were more likely to perceive benefits of the social restriction measures and feel anxiety during the epidemic of COVID-19. Likewise, female, the respondents with better household income and those having good knowledge on preventive measures were more likely to adopt behaviors advised in response to the epidemic of COVID-19. Individual beliefs on benefits of the social restriction measures were also positively associated with the behavioral adoption.

There are a wide array of factors that influence the public behavioral adoption in response to the pandemic of emerging infectious diseases including the government responses, media's portrayal and social culture and values. Compared to the previous studies on the public behavior in response to Severe Acute Respiratory Syndrome (SRAS), H1N1 and MERS epidemic in Hong Kong and other countries, ${ }^{[11-12,}$ 
18-19] our study found behavioral adoption of prevent person-to-person transmission of COVID-19 among the public in Chinese mainland was relatively high. The outbreak of COVID-19 in China occurred around the time of the lunar new year often with massive population travels. When Wuhan city, a center of the epidemic was locked down, number of reported cases increased rapidly and spread nationwide. All Chinese mainland provincial governments launched the highest level of response to the emergent public health event including closure of schools, public services, retail business and restaurants as well as constraints of individual movement and social interactions. The official public media, various social media and community-based approaches widely disseminated the COVID-19 related information and health education. The awareness of the disease severity and benefits of the social restriction measures among the public were high during the lock-down period in China. That is also the period the survey carried out. The study in the Netherland investigated the public response to $\mathrm{H} 1 \mathrm{~N} 1$ at several time points and found the level of knowledge on H1N1 prevention increased over time, while perceived severity, perceived self-efficacy, and intention to comply with preventive measures decreased. ${ }^{[11]}$ Given a high risk of the repeated epidemic of COVID-19, it will be critical to understand the change of attitudes and behavior response to the epidemic of COVID-19 over time to inform strategy sequencing.

This study also suggested that personal protective behaviors, such as wear of face mask when going out, more frequent hand wash and other personal and family hygiene habit were more likely to be widely adopted than social distancing behaviors, which is consistent with findings in other studies. ${ }^{[12,20]}$ In response to the 2009 influenza A H1N1 pandemic, the previous study proposed to combine efforts from both non-pharmaceutical interventions and vaccination program to achieve better preventive effects. ${ }^{\text {[20] }}$ When the vaccine against COVID-19 is not available, approaches adapted to local culture and social value to promoting non-pharmaceutical interventions, particularly social distancing behaviors will be effective to mitigate the burden of COVID-19 in various settings.

Consistent with the other studies on the public responses to emerging infectious diseases, ${ }^{[21-23]}$ we found female and those having better household income and good preventive knowledge were more likely to behave according to health advices. In our study, they were also more likely to perceive the benefits of social restriction measures, which was positively associated with the behavioral adoption. The households with low income may face financial pressure and/ or difficulty due to loss of income or work opportunity during the lock-down period. This vulnerable group may be at a high risk of infection given poor behavioral adoption, even fall in poverty because of the illness. Hence, targeted health policy and other related public policy should pay attention on social vulnerable groups to improve equity in health.

In this study, we found that the people who did not feel anxiety during the epidemic were more likely to adopt the behavior advised that those who feel anxiety. The studies on public psychological behavior responses to SARS in Hong Kong and H1N1 in the Netherland found that moderate level of anxiety were positively associated with adopting preventive behaviors. ${ }^{[24-26]}$ We did not measure the level of anxiety in this study. Our study suggests that psychological support for the public during the epidemic of emerging 
infectious diseases is highly important to guide release of negative emotions and improve healthy behavior.

This study captured the public responses to the pandemic of COVID-19 in China during the most lockdown period and has significant implications for relevant strategies and policies sequencing. There are also several limitations bearing in mind. Due to various constraints during the study period, we used snow-ball sampling methods through the social network of the research team. Despite we received the responses from 31 mainland provinces, the most respondents were from Chongqing municipality where the research team located. In addition, the link of the online survey was distributed through social media. Those who are not able to access to social media due to user unfriendly (e.g. the elders) or infrastructure limitations cannot be reached. Thus, the representation of the respondents may be biased and the generation of the results should be made with caution.

\section{Conclusion}

When the outbreak of COVID-19 in China, the public highly accepted and adopted behaviors advised to slow down the epidemic, although the behavior adoption of personal protective measures is better than social distancing behavior. People with low income or feeling anxiety were less likely to adopt the behavior advised. The policy support should target on the social vulnerable groups. The psychological support should be disseminated through different means, and the consultation should be provided to those who are in need. Further study in a longitudinal design should investigate public reactions to the policy recommendations in response to emerging infectious diseases at different stage in order to adjust relevant strategies in shaping public response.

\section{Abbreviations}

COVID-19: Coronavirus disease 2019; HBM: Health Belief Model; MERS: Middle East Respiratory Syndrome; H1N1: Influenza A; CDC: Centre for Disease Control and Prevention; SRAS: Severe Acute Respiratory Syndrome.

\section{Declarations}

\section{Ethics approval and consent to participate:}

This study obtained the approval by the Ethics Committee of Chongqing Medical University, an approval ID was not provided. All participants were voluntary and provided the consent when they clicked the link to complete the survey online.

\section{Consent for publication:}

Not applicable. 


\section{Availability of data and materials:}

The datasets used and/or analysed during the current study are available from the corresponding author on reasonable request.

\section{Competing interests:}

The authors declare that they have no competing interests.

\section{Funding:}

The study is supported by the Chongqing Science and Technology Association (Yu Science Association [2020]11) and Medical and Social Development Research Center, School of Public Health and Management, Chongqing Medical University (2020YJ05).

\section{Acknowledgements:}

The author thanks all the respondents for participating in the survey and appreciate personal and institutional supports to disseminating the information of the survey.

\section{Authors' Contributions:}

$\mathrm{HX}$ designed and performed the study. $\mathrm{HX}, \mathrm{XZ}, \mathrm{YG}, \mathrm{BW}, \mathrm{QYG}, \mathrm{DKZ}, \mathrm{CLL}, \mathrm{CX}$ and ZT participated in data collection and quality control. QL guided the data analysis, interpretation and manuscript writing. $X Z$ performed the data analyses. XZ, YG, BW and QYG wrote the first draft of the manuscript. All authors read, commented and approved the final manuscript.

\section{References}

1. World Health Organization. Coronavirus disease (COVID-19) outbreak situation. https://www.who.int/emergencies/diseases/novel-coronavirus-2019. Accessed 30 Jun 2020.

2. Li YZ, He H, Chen Q. Research advances on coronavirus disease 2019. J Trop Med. 2020;20(05):581586.

3. Special Expert Group for Control of the Epidemic of Novel Coronavirus Pneumonia of the Chinese Preventive Medicine Association. An update on the epidemiological characteristics of novel Coronavirus Pneumonia (COVID-19). Chin J Viral Dis. 2020; 41(2):139-144.

4. Hui DS, I Azhar E, Madani TA, et al. The continuing 2019-nCoV epidemic threat of novel coronaviruses to global health - The latest 2019 novel coronavirus outbreak in Wuhan, China. Int J 
Infect Dis. 2020;91:264-266. doi:10.1016/j.jij.2020.01.009

5. He Z. What further should be done to control COVID-19 outbreaks in addition to cases isolation and contact tracing measures? BMC Med. 2020;18(1):80. doi:10.1186/s12916-020-01551-8

6. Epidemiology Working Group for NCIP Epidemic Response, Chinese Center for Disease Control and Prevention. The epidemiological Characteristics of an outbreak of 2019 novel Coronavirus diseases (COVID-19) in China. Chin J Epidemiol. 2020,41(2):145-151.

7. World Health Organization. Advice-for-public. https://www.who.int/zh/emergencies/diseases/novelcoronavirus-2019/advice-for-public. Accessed 29 Mar

8. Cheng VCC, Wong SC, To KKW, et al. Preparedness and proactive infection control measures against the emerging Wuhan coronavirus pneumonia in China. J Hosp Infect. doi:

10.1016/j.jhin.2020.01.010

9. Lurie N, Saville M, Hatchett R, et al. Developing Covid-19 Vaccines at Pandemic Speed. N Engl J Med. 2020;382(21):1969-1973. doi:10.1056/NEJMp2005630

10. Rubin EJ, Baden LR, Morrissey S. Audio Interview: New Research on Possible Treatments for Covid19. N Engl J Med. 2020;382(12):e30. doi:10.1056/NEJMe2005759

11. Bults Marloes, Beaujean Desirée, de Zwart Onno, et al. Perceived risk, anxiety, and behavioural responses of the general public during the early phase of the influenza A (H1N1) pandemic in the Netherlands: Results of three consecutive online surveys. BMC Public Health. 2011;11(2):2. doi: 10.1186/1471-2458-11-2

12. Jang WM, Cho S, Jang DH, et al. Preventive Behavioral Responses to the 2015 Middle East Respiratory Syndrome Coronavirus Outbreak in Korea. Int J Environ Res Public Health. 2019;16(12):2161. doi:10.3390/ijerph16122161

13. Tavafian SS, Hasani L, Aghamolaei T, et al. Prediction of breast self-examination in a sample of Iranian women: an application of the Health Belief Model. BMC Womens Health. 2009;9:37. doi: 10.1186/1472-6874-9-37.

14. Dempster NR, Wildman BG, Masterson TL, et al. Understanding Treatment Adherence With the Health Belief Model in Children With Cystic Fibrosis. Health Educ Behav. 2018;45(3):435-443. doi:10.1177/1090198117736346

15. Chen J, Liao Y, Li Z, et al. Determinants of salt-restriction-spoon using behavior in China: application of the health belief model. PLoS One. 2013;8(12):e83262. doi:10.1371/journal.pone.0083262

16. Didarloo A, Nabilou B, Khalkhali HR. Psychosocial predictors of breast self-examination behavior among female students: an application of the health belief model using logistic regression. BMC Public Health. 2017;17(1):861. doi:10.1186/s12889-017-4880-9

17. Skinner CS, Tiro J, Champion VL. The health belief model. Health behavior:

theory, research, and practice. San Francisco, CA: John Wiley \& Sons 2015:75-94. 
18. SteelFisher GK, Blendon RJ, Bekheit MM, et al. The public's response to the $2009 \mathrm{H} 1 \mathrm{~N} 1$ influenza pandemic. N Engl J Med. 2010;362(22):e65. doi:10.1056/NEJMp1005102

19. Lau JT, Yang X, Tsui $H$, et al. Monitoring community responses to the SARS epidemic in Hong Kong: from day 10 to day 62. J Epidemiol Community Health. 2003;57(11):864-870. doi:10.1136/jech.57.11.864

20. SteelFisher GK, Blendon RJ, Ward JR, et al. Public response to the 2009 influenza A H1N1 pandemic: a polling study in five countries. Lancet Infect Dis. 2012;12(11):845-850. doi:10.1016/S14733099(12)70206-2

21. Qi Y, Chen LH, Zhang Li, et al. Research on public cognition, attitude and behavior of new coronavirus pneumonia. J Trop Med. 2020(02).

22. Barber SJ, Kim H. COVID-19 Worries and Behavior Changes in Older and Younger Men and Women [published online ahead of print, 2020 May 19]. J Gerontol B Psychol Sci Soc Sci. 2020;gbaa068. doi:10.1093/geronb/gbaa068

23. Zhong BL, Luo W, Li HM, et al. Knowledge, attitudes, and practices towards COVID-19 among Chinese residents during the rapid rise period of the COVID-19 outbreak: a quick online cross-sectional survey. Int J Biol Sci. 2020;16(10):1745-1752. doi:10.7150/ijbs.45221

24. Leung GM, Ho LM, Chan SK, et al. Longitudinal assessment of community psychobehavioral responses during and after the 2003 outbreak of severe acute respiratory syndrome in Hong Kong. Clin Infect Dis. 2005;40(12):1713-1720. doi:10.1086/429923

25. Leung GM, Lam TH, Ho LM, et al. The impact of community psychological responses on outbreak control for severe acute respiratory syndrome in Hong Kong. J Epidemiol Community Health. 2003;57(11):857-863. doi:10.1136/jech.57.11.857

26. Jones $\mathrm{JH}$, Salathé $\mathrm{M}$. Early assessment of anxiety and behavioral response to novel swine-origin influenza A(H1N1). PLoS One. 2009;4(12):e8032. doi:10.1371/journal.pone.0008032

\section{Table}

Due to technical limitations, table is only available as a download in the Supplemental Files section.

\section{Figures}




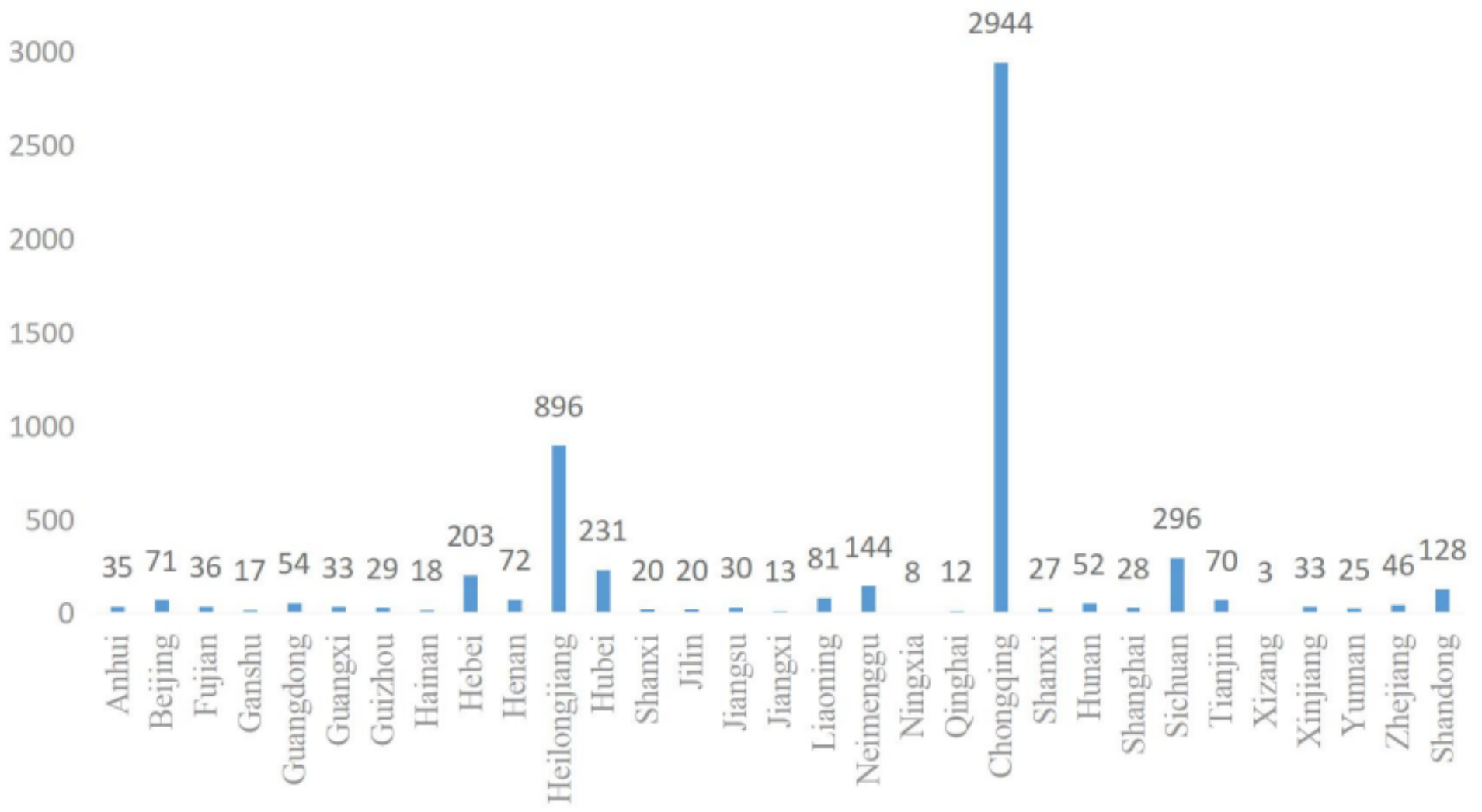

Figure 1

Respondent area distribution map 


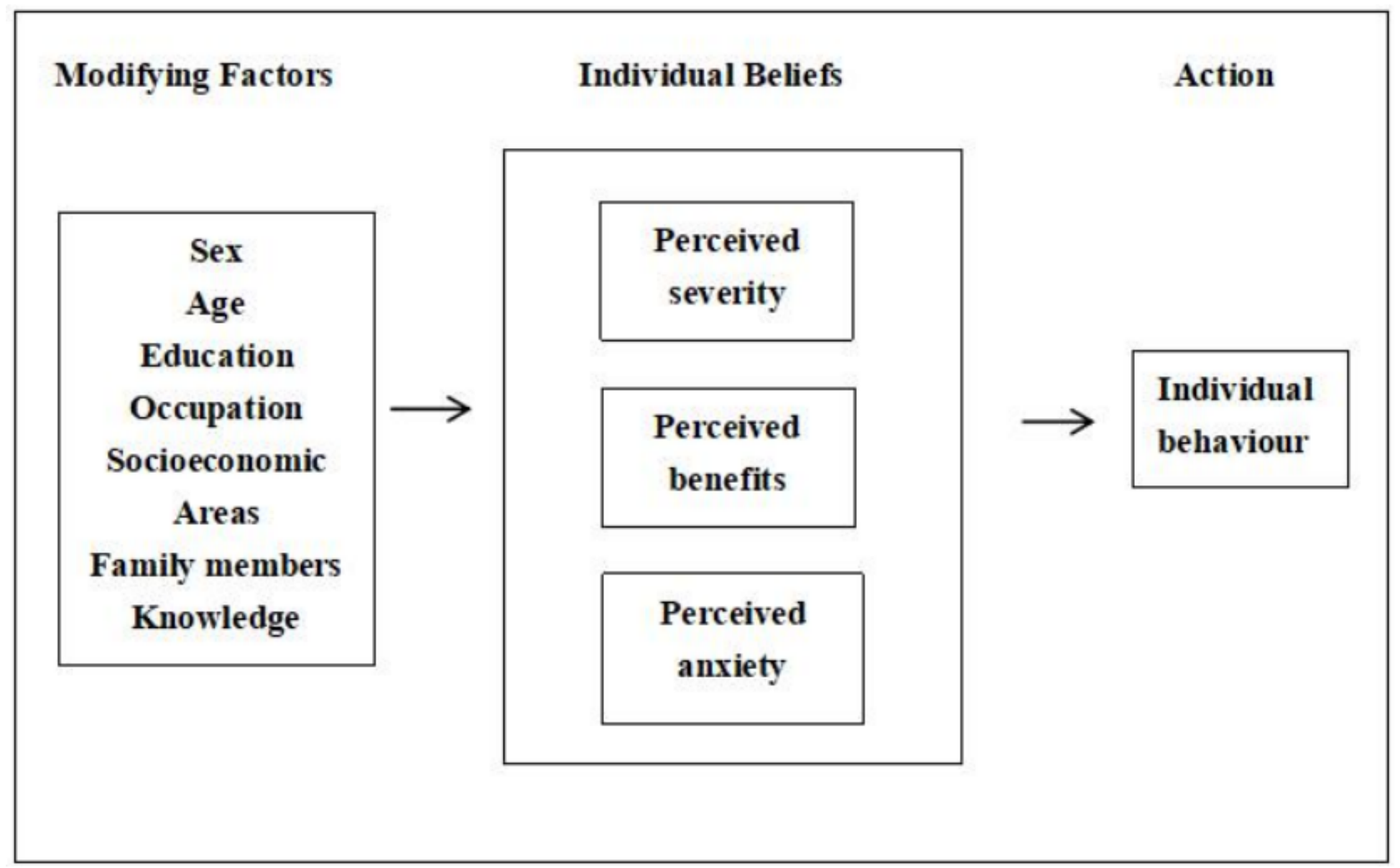

Figure 2

Analysis framework adapted from the Health Belief Model

\section{Supplementary Files}

This is a list of supplementary files associated with this preprint. Click to download.

- Table.pdf 